\title{
Frage zur Lyseindikation bei Lungenembolie
}

\author{
S. Konstantinides
}

\section{Einleitung zur Rubrik}

In dieser Rubrik geben ausgewiesene Experten Antworten auf Ihre Fragen, die sich nicht so einfach aus der Literatur beantworten lassen. Wenn Sie also eine außergewöhnliche Frage haben, auf die Sie schon lange eine Antwort suchen, freuen wir uns auf Ihre Zuschrift an Gabi.Hasenmaile@thieme.de.

\section{Ask the Expert}

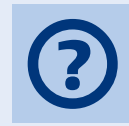

Die S2 Leitlinie „Diagnostik und Therapie der Bein- und Beckenvenenthrombose und der Lungenembolie“ besagt, dass Patienten mit niedrigem Risiko (hämodynamisch stabil, keine rechtsventrikuläre Dysfunktion) wie bei einer Venenthrombose antikoaguliert werden, Patienten mit hohem Risiko systemisch lysiert werden. Patienten mit mittlerem Risiko, d.h. hämodynamisch stabil mit rechtsventrikulärer Dysfunktion, sollen in „ausgewählten Fällen" einer systemischen Thrombolyse zugeführt werden. Gibt es neue Daten bzw. Empfehlungen, wie in solchen Fällen pragmatisch vorgegangen werden kann?

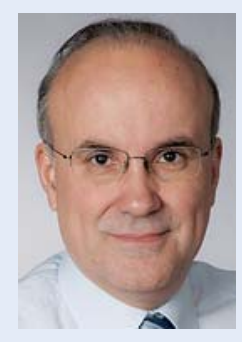

S. Konstantinides

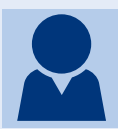

Patienten mit Hochrisiko-Lungenembolie (hämodynamisch instabil, kardiogener Schock) benötigen, abgesehen von kreislaufunterstützenden Maßnahmen, eine sofortige rekanalisierende Therapie in Form einer Thrombolyse, alternativ einer chirurgischen Embolektomie oder kathetertechnischen Intervention. Bei Patienten mit „Nicht-Hochrisiko“-Lungenembolie (LE) wird dagegen eine Thrombolyse nicht routinemäßig empfohlen. Für die meisten dieser Patienten steht die Antikoagulation mit einem niedermolekularen Heparin (NMH) oder Fondaparinux in gewichtsadaptierter Dosierung im Vordergrund der Akuttherapie. Allerdings ist auch ein Teil dieser normotensiven, scheinbar stabilen Patienten durch ein erhöhtes (intermediäres) Todes- und Komplikationsrisiko gefährdet. In der kürzlich publizierten multizentrischen, internationalen, doppel-blind 1:1 randomisierten Pulmonary Embolism Thrombolysis Study (PEITHO) wurde untersucht [1], ob normotensive Patienten mit erhöhten Troponin-Plasmakonzentrationen und Nachweis einer RV Dysfunktion in der transthorakalen Echokardiografie oder Computertomografie von einer frühen thrombolytischen Behandlung profitieren. Von 1006 eingeschlossenen Patienten erreichten 41 Patienten (4,1\%) den primären Endpunkt (hämodynamische Dekompensation/Kollaps oder Tod innerhalb von 7 Tagen). Durch Thrombolyse mit Tenekteplase konnte das Risiko, den primären Endpunkt zu erreichen, um 56\% (2,6 vs. $5,6 \%$ ) reduziert werden (Odds Ratio: 0,44; $95 \%$ Konfidenzintervall: 0,23-0,88). In Übereinstimmung mit der Literatur entwickelten thrombolysierte Patienten jedoch signifikant häufiger schwere ( 6,3 vs. $1,5 \%)$ und intrakranielle Blutungen (2,0 vs. $0,2 \%$ ) [1].

Daher sollten Patienten, die oben genannte Kriterien eines intermediären Risikos bei bestätigter akuter LE erfüllen, nicht routinemäßig primär lysiert werden insbesondere nicht, wenn sie älter als 75 Jahre sind. Vielmehr sollten sie engmaschig über 48 - 72 h überwacht werden und erst bei klinischen Zeichen einer Dekompensation thrombolytisch behandelt werden. Konkret bedeutet das: Diese Patienten werden auf eine „Chest Pain Unit“, „Coronary Care Unit“ oder „Intermediate Care Unit“ bzw. sogar auf eine Intensivstation aufgenommen und überwacht. Sie erhalten dort entweder eine Infusion von unfraktioniertem Heparin (in aPTT-angepasster Dosis) oder ein niedermolekulares Heparin, z. B. Enoxaparin oder Tinzaparin oder Fondaparinux als ein- bzw. zweimal tägliche subkutane Injektion. Die empfohlenen Heparindosierungen sind der „Diagnostik und Therapie der Bein- und Beckenvenenthrombose und der Lungenembolie“ zu entnehmen [2]. 48 - 72 h später kann bei hämodynamischer Stabilität auf einen Vitamin-K-Antagonisten oder ein neues orales Antikoagulans (Dabigatran, Rivaroxaban oder Apixaban) umgestellt werden. Falls es dagegen in diesem Zeitraum zu einer klinischen Dekompensation 
kommt, sollte eine thrombolytische Therapie durchgeführt werden (z. B. Alteplase in einer Dosis von $100 \mathrm{mg}$ i.v. über $2 \mathrm{~h}$, davon die ersten $10 \mathrm{mg}$ als Bolusinjektion). Im Anschluss an die Thrombolyse ist die Heparininfusion oder -injektion über $48 \mathrm{~h}$ fortzusetzen; erst danach kann auf eine orale Antikoagulation umgestellt werden.

Die Ergebnisse von 2 kleinen, randomisierten Pilotstudien lassen annehmen, dass durch eine niedrigdosierte thrombolytische Therapie (halbierte Alteplase-Standarddosis, d.h. $50 \mathrm{mg}$ oder weniger) das Auftreten einer erneuten Lungenembolie und/oder eine persistierende pulmonale Hypertonie reduziert werden könnten - ohne das Risiko signifikanter Blutungsereignisse $[3,4]$. Diese „Dosisadaptierung“ ist allerdings zum jetzigen Zeitpunkt nicht offiziell zugelassen und daher nicht allgemein zu empfehlen.

Bei zu erwartendem hohem Blutungsrisiko unter Thrombolyse (primär bedingt durch ein Alter $>75$ Jahre, aber auch z.B. durch postoperativen Status oder schweres Trauma) können alternativ operative oder interventionelle Rekanalisationsverfahren angewendet werden. Das Konzept eines neuen Hybridverfahrens, einer ultraschallverstärkten, katheterassistierten, niedrigdosierten (sog. pharmakomechanischen) Thrombolyse, wurde kürzlich in 2 multizentrischen klinischen Studien, einer randomisierten Studie an 59 Patienten in Europa [5] und einer einarmigen Studie an 150 Patienten in den USA (NCT01513759; vorgestellt, aber noch nicht publiziert) untersucht. In beiden relativ kleinen Studien konnte die Sicherheit dieses Regimes, insbesondere das Fehlen intrakranieller Blutungen, bei erhaltener Wirksamkeit der lokal applizierten Lyse gezeigt werden.

Interessenkonflikt: S. Konstantinides: Honorare von Boehringer Ingelheim für wissenschaftliche Vorträge und wissenschaftliche Beratung.

\section{Korrespondenzadresse}

Univ.-Prof. Dr. Stavros Konstantinides

Professur Clinical Trials und

Ärztlicher Direktor

Centrum für Thrombose und Hämostase (CTH)

UNIVERSITÄTSMEDIZIN der Johannes Gutenberg-

Universität Mainz Gebäude 403

Langenbeckstraße 1

55131 Mainz

E-Mail: Stavros.Konstantinides@unimedizin-mainz.de

www.cth-mainz.de

\section{Literatur}

1 Meyer G, Vicaut E, Danays T et al. Fibrinolysis for patients with intermediate-risk pulmonary embolism. N Engl J Med 2014; 370: $1402-1411$

2 Interdisziplinäre S2-Leitlinie. Diagnostik und Therapie der Bein- und Beckenvenenthrombose und der Lungenembolie. Vasa 2010; 39 (Suppl 78): 1 - 39

3 Sharifi M, Bay C, Skrocki L et al. Moderate pulmonary embolism treated with thrombolysis (from the "MOPETT" Trial). Am J Cardiol 2013; 111: 273-277

4 Wang C, Zhai Z, Yang Y et al. Efficacy and safety of low dose recombinant tissue-type plasminogen activator for the treatment of acute pulmonary thromboembolism: a randomized, multicenter, controlled trial. Chest 2010; 137: 254-262

5 Kucher N, Boekstegers P, Muller OJ et al. Randomized, controlled trial of ultrasound-assisted catheter-directed thrombolysis for acute intermediate-risk pulmonary embolism. Circulation 2014; 129: 479-486 